\title{
Labyrinthine fenestration for stapes fixation in chronic ear disease others than otosclerosis
}

\author{
Yannick Zaugg $\cdot$ Thomas Linder
}

Received: 3 February 2014 / Accepted: 25 May 2014 / Published online: 5 June 2014

(C) Springer-Verlag Berlin Heidelberg 2014

\begin{abstract}
The objective of this study is to assess the results of labyrinthine fenestration for fixed stapes in chronic ear disease. Using a prospective database, preand postoperative audiometric data from patients undergoing labyrinthine fenestration for fixation of the stapes in chronic ear disease others than otosclerosis between 2002 and 2012 were evaluated. Twenty-three labyrinthine fenestrations in chronic ear disease were performed (17 malleo-stapedotomies, 4 incus-stapedotomies, 1 neomalleus-stapedotomy, 1 TORP-stapedotomy). Overall, the mean short-term ( 2 months) and long-term (42 months) postoperative air-bone gap $(0.5-3 \mathrm{kHz})$ were 17.5 and $16.5 \mathrm{~dB}$, respectively; long-term air-bone gap of $<20 \mathrm{~dB}$ was obtained in $73 \%$ of patients. There was no significant difference in air-bone gap closure between tympanosclerotic and post inflammatory osteogenic fixation of the stapes $(p=0.267)$. Hearing benefit success using the 'Belfast rule of the thumb' was achieved in $48 \%$. Normal bilateral hearing was achieved in $17 \%$ and bilateral symmetric hearing impairment in $26 \%$. Only in $4 \%$, bone conduction worsened by more than $5 \mathrm{~dB}$. Labyrinthine fenestration is an option in selected cases of stapes fixation in chronic ear disease and provides hearing gain without significant risk for sensorineural hearing loss. In those already selected cases, hearing benefit success 'Belfast rule of the thumb'
\end{abstract}

Y. Zaugg $(\bowtie)$

Department of ENT, Head and Neck Surgery, Centre Hospitalier

Universitaire Vaudois, Avenue du Bugnon 21, 1011 Lausanne,

Switzerland

e-mail: yannick.zaugg@chuv.ch

T. Linder

Department of ENT, Head and Neck Surgery, Luzerner

Kantonspital, 6006 Luzern, Switzerland

e-mail: Thomas.linder@luks.ch is achieved only in half of the cases. This and the possible alternatives, should therefore be discussed preoperatively.

Keywords Chronic ear disease - Stapedotomy · Stapes . Tympanosclerosis · Ossiculoplasty

\section{Introduction}

The management of stapes fixation in chronic ear disease remains controversial and is more challenging than in otosclerosis patients. Whereas some surgeons are reluctant to perform surgery, claiming a high risk of sensorineural hearing loss and bad long-term results, others believe that significant benefit can be achieved with surgery. The basic principles of treatment are restoration of footplate mobility or labyrinthine fenestration (stapedotomy, stapedectomy, third window approach). Mobilization of the stapes was generally considered as the first option and remains the technique of choice for several otologist $[1,2]$. If mobilization fails to improve hearing, some are reluctant to open the labyrinth because of the risk of inner ear damage [3] and propose a hearing aid. Others choose labyrinthine fenestration either as salvage surgery after insufficient mobilization attempts [1, 2, 4] or opt for labyrinthine fenestration as primary choice [5-7], claiming that mobilization itself carries the same risks for the inner ear and that refixation can occur. With the improvements in surgical techniques, instrumentation and prosthesis design, labyrinthine fenestration has become a preferred option for selected patients with chronic ear disease and stapes fixation. Selection criteria for fenestrations include patients with a closed cavity, well ventilated middle ear free of recurrent infections for at least 6 months. The current study reviewed our results of labyrinthine fenestration for stapes fixation in chronic ear disease. 


\section{Materials and methods}

In a prospective statistical database (www.innoforce.com), collecting all patients undergoing otological surgery since 2002 , a retrospective analysis of all labyrinthine fenestrations performed for fixation of the stapes in chronic ear disease was performed. Cases of otosclerosis were excluded. Stapes footplate fixation was confirmed during surgery by palpation. Retrospective analysis of the causes of stapes fixation was performed in order to differentiate between tympanosclerosis and post inflammatory osteogenic fixation [8]. The study was approved by the institutional review board.

The surgical technique and the pre- and postoperative audiometric data for all patients were evaluated. Audiometric data were analyzed according to the recommendations of the Committee on Hearing and Equilibrium [9]. We used the four pure tone averages (PTA) for bone and air thresholds at $0.5,1,2$, and $3 \mathrm{kHz}$. The air-bone gap (ABG) was expressed as the four PTA for air conduction minus the same average for bone conduction.

For short-term results, the preoperative audiogram was compared to the first available postoperative audiogram. In order to assess the functional benefit, short-term PTA were plotted in the Glasgow benefit plot [10]. As the 'Belfast rule of the thumb' provides a significant degree of correlation with the patients' estimate of benefit, hearing outcome was also calculated with this rule. The 'Belfast rule of the thumb' is fulfilled when postoperative PTA $(0.5-4 \mathrm{kHz})$ is less or equal to $30 \mathrm{~dB}$ for the operated ear or if the interaural difference is reduced to less or equal of $15 \mathrm{~dB}$ [11]. For patients with a follow-up of more than 1 year, the long-term results were compared using the preoperative and the last available postoperative audiogram. Short- and long-term audiometric results were compared as well. Statistical analysis consisted of Wilcoxon signed-ranks test for comparison of quantitative variables of two paired groups and Mann-Whitney $U$ test for comparison of the averages of two independent groups of samples; $p<0.05$ was considered to be statistically significant.

\section{Results}

\section{Surgical techniques}

Labyrinthine fenestration was performed in 23 selected patients with chronic ear disease and stapes fixation, others than otosclerosis, between 2002 and 2012. There were 14 females and 9 males. The average age was 45 years (range 11-72 years).

A calibrated opening into the stapes footplate is preferred over total stapedectomy to reduce the risk of inner ear damage [12]. Therefore a stapedotomy of $0.6 \mathrm{~mm}$ in diameter was performed in all patients. Drill, lasers (Erbium:YAG or KTP)
Table 1 Type of reconstruction used and their respective postoperative air-bone gap

\begin{tabular}{lrl}
\hline Type of reconsturction & \multicolumn{1}{c}{$n$} & Post-op ABG dB [SD] \\
\hline Malleo-stapedotomy & 17 & $17.2(7.7)$ \\
Incus-stapedotomy & 4 & $14.7(5.9)$ \\
TORP-stapedotomy & 1 & 17.5 \\
Neo-malleus-stapedotomy & 1 & 35.5 \\
\hline
\end{tabular}

$n$ number of patient

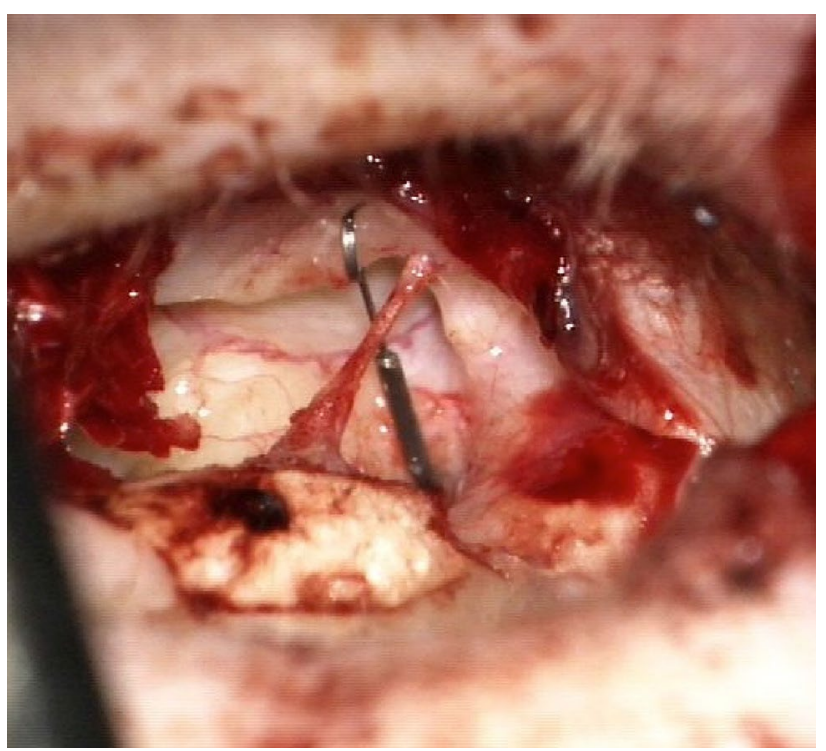

Fig. 1 Intra-operative view of a malleo-stapedotomy with a Fisch titanium stapes piston ( $0.4 \mathrm{~mm}$ diameter) attached to the upper malleus handle

and manual perforators were used alone or in combination in 22,70 and $91 \%$ respectively. The types of reconstructions are listed in Table 1 . The choice was dictated by the intraoperative findings. When incus and malleus were present and mobile, an incus-stapedotomy with a $0.4 \mathrm{~mm}$ Fisch titanium stapes piston (Karl Storz, Tuttlingen, Germany) was performed. When incus or the head of malleus were fixed, they were removed leaving a malleus handle. Then a malleo-stapedotomy with a $0.4 \mathrm{~mm}$ Fisch titanium stapes piston (Karl Storz, Tuttlingen, Germany) was performed (Fig. 1). The malleus handle was absent in two patients. In the first patient, a neo-malleo-stapedotomy, in a 2-stage procedure was performed. After inserting a titanium neomalleus (Karl Storz, Tuttlingen, Germany) into the reconstructed ear drum, a neomalleo-stapedotomy was performed 6 months later [13]. In the second patient without a malleus handle, a $0.6 \mathrm{~mm}$ stapedotomy was performed, covered with perichondrium and a TORP was inserted (Fig. 2). In all cases, the oval window niche was sealed with two to three small pieces of soft tissue and a few drops of patient's own blood. 


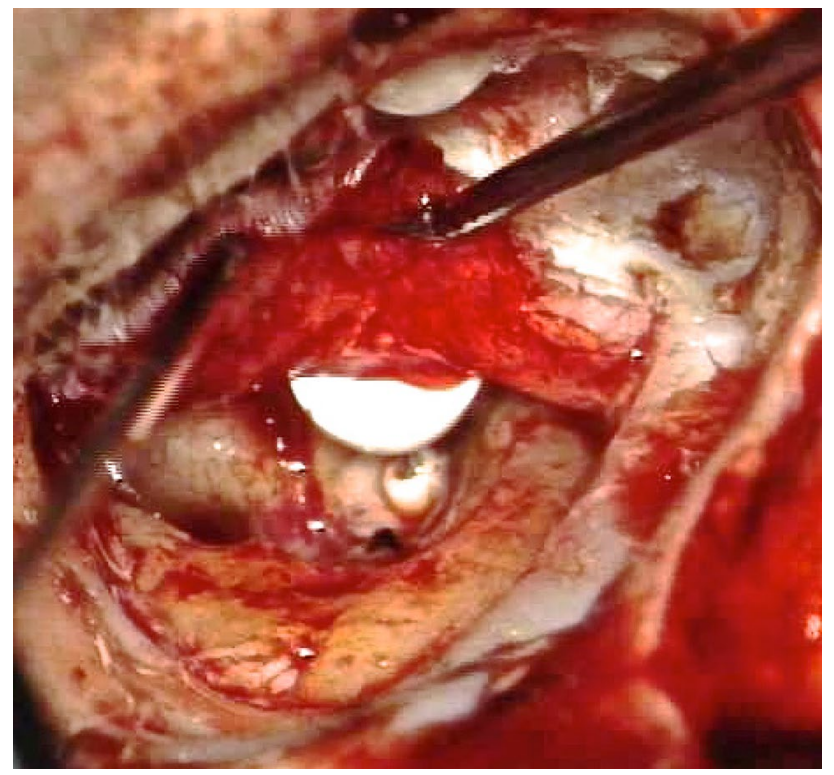

Fig. 2 Intra-operative view of a stapedotomy with a TORP in an open cavity

In cases of mild or extensive tympanosclerosis the cause of fixation presented no particular problem. Fourteen patients were classified as such. The cases of otosclerosis or suspected otosclerosis with a conductive hearing loss with normal tympanic membrane and no previous surgery were already excluded. For the nine other patients the causes of fixation was more subtule. At the time of surgery it is not always possible to visualize through the external ear canal the exact location and morphology of the fixing lesion in the attic. Using the following criteria described by Shea [8] bony fixation involving the superstructure of the stapes, or when stapes fixation occurred in an ear that had undergone relatively recent surgery were the stapes was clearly mobile, five patients could be classified as postinflammatory osteogenic fixation. The last four patients couldnot be classified because details of stapes fixation were not sufficiently documented in the database.

\section{Audiometry results}

Short-term results were available for all 23 stapedotomy with a mean follow-up of 2 months (range 0.7-5.8 months).

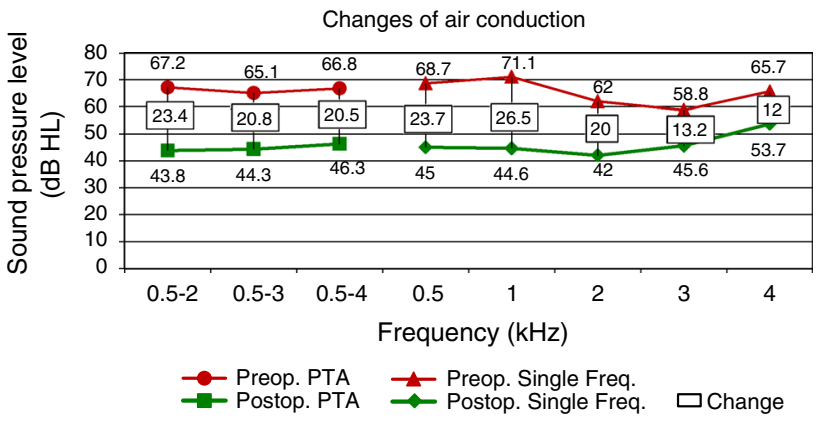

Fig. 3 Line graph showing changes in air conduction thresholds following stapedotomy for fixed stapes in chronic ear disease. Pre-op preoperative, PTA pure tone average, freq frequency, Post-op postoperative [2 months $(0.7-5.8)]$

Postoperative air-bone gap: the pre- and short-term postoperative $\mathrm{ABG}$ are reported in Table 2. This demonstrated a mean preoperative $\mathrm{ABG}(0.5-3 \mathrm{kHz})$ of $33.8 \mathrm{~dB}$ and a mean short-term postoperative $\mathrm{ABG}$ of $17.5 \mathrm{~dB}$. Overall, $74 \%$ of patients remained within $20 \mathrm{~dB}$ and $17 \%$ within $10 \mathrm{~dB}$. Comparison of the short-term postoperative ABG between the tympanosclerotic fixation of the stapes $(17.6 \mathrm{~dB})$ and the postinflammatory osteogenic fixation (20.1) was not statistically significant $(p=0.267)$.

Air conduction threshold: short-term changes in PTA and air conduction for single frequency are plotted in Fig. 3. The mean PTA gain $(0.5-3 \mathrm{kHz})$ was $20.8 \mathrm{~dB}$. Comparison of the short-term postoperative PTA between the tympanosclerotic fixation of the stapes and the postinflammatory osteogenic fixation was not statistically significant $(p=0.18)$.

Bone conduction threshold: change between pre- and short-term postoperative bone conduction is shown in Fig. 4 . The postoperative bone conduction thresholds were within $5 \mathrm{~dB}$ of preoperative values in $74 \%$, over the frequencies $0.5-3 \mathrm{kHz}$. In $4 \%$ of all patients, bone conduction worsened by more than $5 \mathrm{~dB}$. In $22 \%$, an improvement (i.e. over-closure) was documented. There was no dead ear.

Glasgow rule of the thumb: normal bilateral hearing or symmetrical hearing was achieved in $48 \%$ of the patients.

Glasgow benefit plot: Fig. 5 shows the preoperative and short-term postoperative Glasgow benefit plot $(0.5-3 \mathrm{kHz})$. Normal bilateral hearing was achieved in four patients
Table 2 Pre- and short-term postoperative air-bone gap result

$A B G$ air-bone gap, Preop preoperative, Post-op postoperative [2 months $(0.7-5.8)]$

\begin{tabular}{llllllll}
\hline & $0.5-3 \mathrm{kHz}$ & $0.5-4 \mathrm{kHz}$ & $0.5 \mathrm{kHz}$ & $1 \mathrm{kHz}$ & $2 \mathrm{kHz}$ & $3 \mathrm{kHz}$ & $4 \mathrm{kHz}$ \\
\hline Pre-op ABG (mean; dB) & 33.8 & 36.3 & 45.0 & 42.8 & 22.4 & 25.1 & 34.8 \\
Post-op ABG (mean; dB) & 17.5 & 19.6 & 24.3 & 21.5 & 8.5 & 15.8 & 23.9 \\
Pts with ABG closure & & & & & & & \\
$\leq 10 \mathrm{~dB}(\%)$ & 17 & 17 & 22 & 22 & 78 & 48 & 17 \\
$\leq 20 \mathrm{~dB}(\%)$ & 74 & 61 & 39 & 65 & 96 & 74 & 57 \\
\hline
\end{tabular}


Fig. 4 Assessment of the changes of bone conduction. Improvement or aggravation of more than $5 \mathrm{~dB}$ was considered

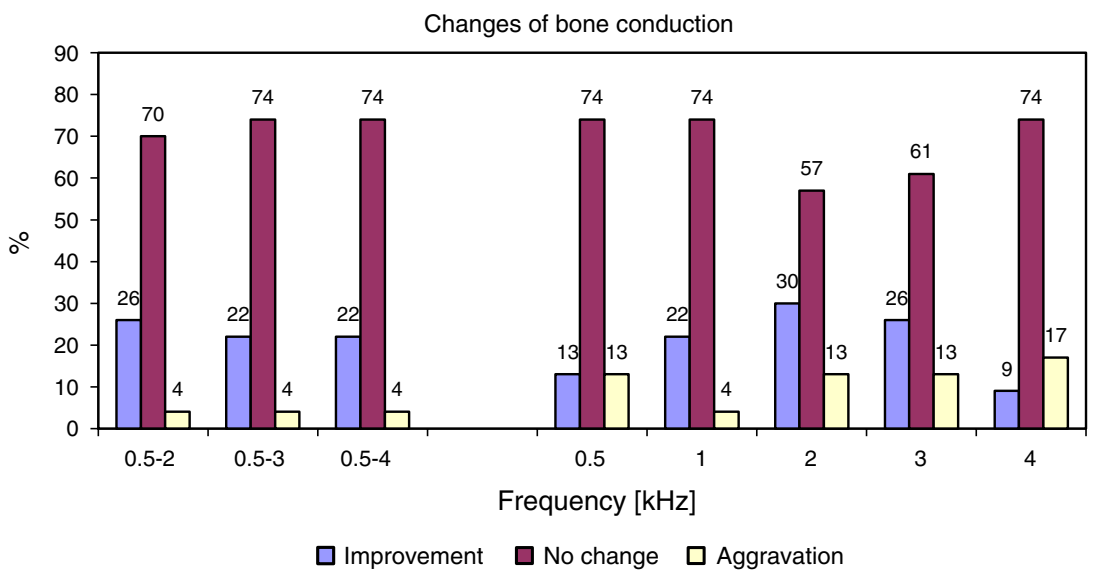

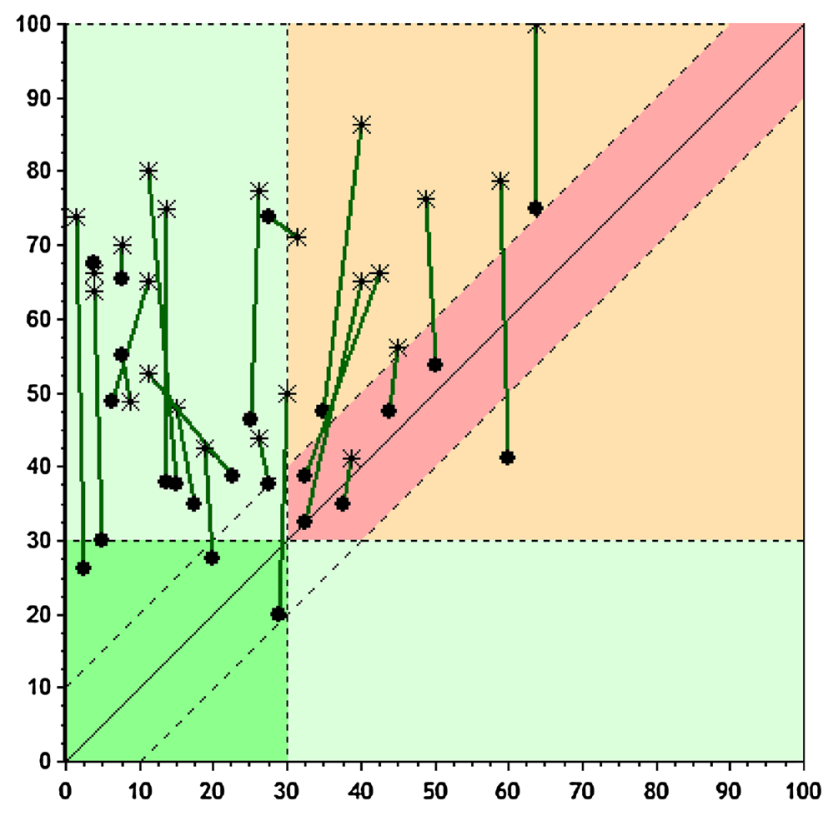

Fig. 5 Glasgow benefit plot: short-term postoperative change in pure tone average $(0.5-3 \mathrm{kHz})$ thersholds (asterisk preoperative, circle short-term postoperative [2 months $(0.72-5.8)$ ]

(17\%) and bilateral symmetric hearing impairment in six patients $(26 \%)$. Globally a benefit was achieved in $43 \%$.

Long-term results were available for 15 out of 23 patients with a mean follow-up of 42 months (range 12100 months). Hearing results were stable over time. There were no statistically significant difference between the short-term and the long-term postoperative PTA $(p=0.69)$ or ABG $(p=0.89)$ (Table 3$)$.

\section{Discussion}

Stapes fixation in chronic ear disease is fortunately an infrequent intraoperative finding. Shea in 1977 reviewed
Table 3 Short- and long-term air-bone gap results

\begin{tabular}{|c|c|c|}
\hline & $\begin{array}{l}\text { Shortterm } \\
\qquad(n=23)\end{array}$ & $\begin{array}{l}\text { Longterm } \\
\qquad(\mathrm{n}=15)\end{array}$ \\
\hline Pre-op $A B G$ (mean; $d B)$ & 33.8 & 33.2 \\
\hline Post-op ABG (mean; dB) & 17.5 & $p=0,89 \stackrel{16.5}{\longrightarrow}$ \\
\hline $\begin{array}{l}\text { Pts with ABG closure } \\
\quad \leq 10 \mathrm{~dB}(\%) \\
\leq 20 \mathrm{~dB}(\%)\end{array}$ & $\begin{array}{l}17 \\
74\end{array}$ & $\begin{array}{l}27 \\
73\end{array}$ \\
\hline
\end{tabular}

his 1,502 surgeries for chronic otitis media and found an incidence of stapes fixation in 112 patients or $7.5 \%$ overall [8]. Within this subgroup $62 \%$ were due to tympanosclerosis. Since tympanoclerosis is an "end-stage" disease, the ear is usually dry and the non-affected mucosa can be quite healthy. Involvement of the tympanic cavity and the ossicles typically leads to severe hearing loss with an air-bone gap of $40 \mathrm{~dB}$ or more. Shea described another cause of stapes fixation which he termed "postinflammatory osteogenic fixation". Analyzing his 1,502 middle ear surgeries for chronic otitis media, he found 19 cases (17\% of the stapes fixation) with such a rigid stapes fixation as a consequence of chronic ear disease. All our patients were selected to undergo a stapes fenestration once they fulfilled the criteria of a dry, ventilated middle ear with preferred presence of an incus and/or malleus for prosthesis fixation. Patients with thickened or markedly retracted tympanic membranes, severe blunting of the drum after previous surgeries or unhealthy middle ear mucosa were not considered for surgery. They were fitted with hearing aids or underwent implantation of bone conducting hearing devices.

The majority of our patients underwent a malleo-stapedotomy, mainly because the ossicular chain was interrupted by the underlying chronic pathology or previous surgery, or had to be partially resected to restore mobility of the malleus handle. In case of tympanosclerosis a simultaneous 
fixation of incus and/or malleus in the attic and the stapes varies between 10 and $47 \%[1,14]$ indicating the need for incus and malleus head removal. Luckily the malleus handle and the tensor tendon can be maintained most of the time providing a crimping site for the piston.

The results showed that stapedotomy ensured a shortterm and long-term hearing gain. The mean short-term PTA gain was $20.8 \mathrm{~dB}$ and $73 \%$ reached a postoperative ABG within $20 \mathrm{~dB}$. These results are less satisfactory than the results of stapes surgeries in otosclerosis with a healthy and well-ventilated middle ear [15]. However, our results correlate well with other series. Vincent, Teufert and Stankovic describe postoperative ABG within $20 \mathrm{~dB}$ with fenestration in 70, 60 and $50 \%$ of the cases, respectively [2, 5, 16]. Sensorineural hearing impairment was recognized in $4 \%$ of our patients with no severe hearing loss. The range reported in the literature varies from 0 to $27 \%,<10 \%$ in recent larger series $[2,5,14,17,18]$. For stapes mobilization the risk of sensorineural hearing loss has been reported from 0 to $17 \%$ [2, 17, 18].

Quite surprisingly, hearing improvement and postoperative air-bone gap seem not to differ between mobilization or fenestrations procedures $[2,4,19]$. Some authors report better results after stapedectomy, whereas others favor mobilization to be more effective [18]. For example Smyth [17] obtained closure of the ABG within $10 \mathrm{~dB}$ in $85 \%$ with stapedectomy versus $54 \%$ with mobilization and in contrast Kinney [18] reported an ABG closure within $10 \mathrm{~dB}$ in $57 \%$ with mobilization versus $39 \%$ with stapedectomy. Comparison between different studies regarding the outcome of stapes mobilization varies since the amount of sclerotic plaque removal is never described or quantified and the initial and final mobility of the stapes is judged clinically and depends on the experience of the surgeon. In this study there was no significant difference in postoperative ABG between tympanosclerotic and post inflammatory fixation of the stapes. Shea described that in cases of post inflammatory fixation of the stapes postoperative ABGs were within $20 \mathrm{~dB}$ in $91 \%$ of the cases of stapedectomy (10 out of 11) but only in $21 \%$ ( 2 out of 7 ) of the cases of stapes mobilization [8]. In case of post inflammatory fixation, fenestration should be preferred to mobilization, but strong evidences are lacking to make recommendation.

According to our data the results seem to be stable over time. This is in correlation with Teufert and Giddings [2, 4]. The latter described a decline from 5 to 10 years, but the difference was not statistically significant. Stankovic describes a slight hearing deterioration with time [16]. Our median follow-up of 42 months ( $31 / 2$ years) is probably not sufficient enough to predict true long-term results.

Whereas PTA and ABG improved considerably following surgery, the overall hearing benefit calculated by the 'Belfast rule of the thumb' or the Glasgow benefit plot was achieved only in about half of the cases (48 and $43 \%$ ). We caution the statement by Querat "labyrinthine fenestration in patients with tympanosclerotic stapes fixation appears to provide useful functional results" [6]. Vincent et al. also used the Glasgow benefit plot and described postoperative bilateral normal hearing in $22 \%$; however, did not further discuss this finding.

Results of ossiculoplasties compete with the functional gain provided by bone anchored hearing aids and active middle ear implants for mixed hearing loss [20]. Even vibroplasty onto the round window membrane may be performed in cases of a fixed stapes footplate [21-23]. Surgery with labyrinthine fenestration has to be scheduled carefully, providing a well-ventilated middle ear space and the drum in a stable position.

\section{Conclusion}

Our results reveal that labyrinthine fenestration is an option in selected cases of stapes fixation in chronic ear disease and provides hearing gain without significant risk for sensorineural hearing loss. The overall benefit is less favorable than in otosclerosis patients and depends on the contralateral ear as well. Adhering to these stricter rules a real success is only achieved in half of the cases. These considerations and the alternatives should be discussed preoperatively with the patient.

Conflict of interest None.

\section{References}

1. Tos M, Lau T, Arndal H, Plate S (1990) Tympanosclerosis of the middle ear: late results of surgical treatment. J Laryngol Otol 104:685-689

2. Teufert KB, De La Cruz A (2002) Tympanosclerosis: long-term hearing results after ossicular reconstruction. Otolaryngol Head Neck Surg 126:264-272

3. Tsuzuki K, Yanagihara N, Hinohira Y, Sakagami M (2006) Tympanosclerosis involving the ossicular chain: mobility of the stapes in association with hearing results. Acta Otolaryngol 126:1046-1052

4. Giddings NA, House JW (1992) Tympanosclerosis of the stapes: hearing results for various surgical treatments. Otolaryngol Head Neck Surg 107:644-650

5. Vincent R, Oates J, Sperling NM (2002) Stapedotomy for tympanosclerotic stapes fixation: is it safe and efficient? A review of 68 cases. Otol Neurotol 23:866-872

6. Querat C, Richard C, Martin C (2012) Labyrinthine fenestration for tympanosclerotic stapes fixation. Eur Ann Otorhinolaryngol Head Neck Dis 129:297-301

7. Martin H, Martin C (1979) Typanosclerosis and labyrinthine trephination. Results. Ann Otolaryngol Chir Cervicofac 96:307-314

8. Shea MC Jr (1977) Postinflammatory osteogenic fixation of the stapes. Laryngoscope 87:2056-2065 
9. Committee on Hearing and Equilibrium (1995) Guidelines for the evaluation of results of treatment of conductive hearing loss. Otolaryngol Head Neck Surg 113:186-188

10. Browning GG (1993) Reporting the benefits from middle ear surgery using the Glasgow benefit plot. Am J Otol 14:135-140

11. Smyth GD, Patterson CC (1985) Results of middle ear reconstruction: do patients and surgeons agree? Am J Otol 6:276-279

12. Fisch U (1982) Stapedotomy vs stapedectomy. Am J Otol 4:112-117

13. Fisch U, May JS, Linder T (2008) Tympanoplasty, mastoidectomy, and stapes surgery, 2nd edn. Thieme, Stuttgart

14. Albu S, Babighian G, Trabalzini F (2000) Surgical treatment of tympanosclerosis. Am J Otol 21:631-635

15. Vincent R, Sperling NM, Oates J, Jindal M (2006) Surgical findings and long-term hearing results in 3,050 stapedotomies for primary otosclerosis: a prospective study with the otology-neurotology database. Otol Neurotol 27:S25-S47

16. Stankovic MD (2009) Hearing results of surgery for tympanosclerosis. Eur Arch Otorhinolaryngol 266:635-640
17. Smyth GD (1972) Tympanosclerosis. J Laryngol Otol 86:9-14

18. Kinney SE (1978) Postinflammatory ossicular fixation in tympanoplasty. Laryngoscope 88:821-838

19. Emmett JR, Shea JJ (1978) Surgical treatment of tympanosclerosis. Laryngoscope 88:1642-1648

20. Verhaert N, Desloovere C, Wouters J (2013) Acoustic hearing implants for mixed hearing loss: a systematic review. Otol Neurotol 34:1201-1209

21. Beltrame AM, Martini A, Prosser S, Giarbini N, Streitberger C (2009) Coupling the Vibrant Soundbridge to cochlea round window: auditory results with mixed hearing loss. Otol Neurotol 30:194-201

22. Schwab B, Salcher RB, Maier H, Kontorinis G (2012) Oval window membrane vibroplasty for direct acoustic cochlear stimulation: treating severe mixed hearing loss in challenging middle ears. Otol Neurotol 33:804-809

23. Pau HW, Just T (2010) Third window vibroplasty: an alternative in surgical treatment of tympanosclerotic obliteration of the oval and round window. Otol Neurotol 31:225-227 